\title{
The analysis of artifacts generated in Microwave near-field breast cancer detection
}

\author{
Zhongling $\mathrm{Han}^{1,2}$, a, Zhifu Tao ${ }^{3}$, Meng Yao ${ }^{1}$ \\ ${ }^{1}$ College of information science and technology, East China Normal University, Shanghai 200041, \\ China \\ ${ }^{2}$ College of information science and technology, Shihezi University, Shihezi 832000, China \\ ${ }^{3}$ Department of Electronics, Suzhou Vocational University, Suzhou 215104, China \\ a316685831@qq.com
}

Keywords: breast tumor; microwave near-field detection; imaging artifacts.

\begin{abstract}
Firstly, this paper analyzes the relationship between the detection data and the target tissue interface, and inverse the target image by microwave near-field detection data according to this relationship. According to the characteristics of microwave detection, this paper puts forward a practical inversion method, which is comparison with CT imaging method. Finally, it studies the artifacts in inversion imaging.
\end{abstract}

\section{Introduction}

For a long time, microwave engineers have dreamed using nonionizing electromagnetic waves to image the human body in order to detect cancers ${ }^{[1-4]}$. Over the past several years, significant progress has been made towards making this dream a reality for breast cancer detection. Breast tumors have electrical properties at microwave frequencies that are significantly different than those of healthy breast tissues ${ }^{[5]}$. The liquid content of tumor tissue is much higher than that of the surrounding normal tissues. The conductivity and dielectric constant is different from the surrounding normal tissues. In the process of propagation, electromagnetic waves would occur reflection and refraction on the surface of the medium boundary, such as, and the transmission path of electromagnetic wave would be changed. Based on this feature, we detect and locate breast tumor by measured the transmission distance and the energy of microwave reflection signal in different medium. We use slot step frequency modulation method to obtain signals in actual breast tumor target information acquisition system ${ }^{[6]}$. The bandwidth of slop step frequency modulation emission signal is $200 \mathrm{MHz}$, intermediate frequency is $1.575 \mathrm{GHz}$, and scanning period is $1 \mathrm{~ms}$. The transmitting signal reaches the receiving antenna and mixer after being reflected by breast tumor, while the other part directly gets to the mixer through transmitting antenna coupling networks. The course difference of two parts, which can be deduced from zero-IF, the output of the mixer, is used to determine the distance between the antenna and the tumor.

\section{A comparative approach CT imaging and microwave near-field imaging}

The actual breast tissues are very complex. Different interface have reflection (back wave) and refraction. These signals, which processed by difference frequency between the original signal and the back wave, have many frequency components. We assume that the distribution of dielectric constants of internal breast is $\mu(x, y)$, and $(x, y)$ is the coordinate relative the center point. Obviously, $\mu(x, y)$ is bounded, and the back wave (reflection) occurs in the position which dielectric constant distribution is discontinuous. As shown in Figure 1, $O$ is origin coordinate, $P_{i}$ is detection point and $Q_{x}$ is the interface between different tissues, which also is position with discontinuous dielectric constants. Reflection data obtained by test point $P_{i}$ is that the center of the circle, accumulate all possible back waves on the circular arc with the center $\mathrm{Pi}$ and the radius $P_{i} Q_{x}$. It is proportional to the integral of $\mu(x, y)$ along the $L_{i}$. 


$$
\begin{aligned}
& T_{p i}=a_{i} \mu\left(r_{x}^{i}\right) \\
& \mu\left(r_{x}^{i}\right)=\int_{L i} \mu(x, y) d l
\end{aligned}
$$

Where $a_{i}$ is coefficient and $r_{x}^{i}$ is $\overrightarrow{\boldsymbol{P}_{\boldsymbol{i}} \mathbf{Q}_{x}} \cdot L_{i}$ is a circular arc with center Pi and radius $\left\|r_{x}^{i}\right\|$. Because the back wave outside the model can be ignored, the integral can be simplified as

$$
\mu\left(r_{x}^{i}\right)=\int_{0}^{2 \pi} \mu(x, y) \times r_{x}^{i} d \theta
$$

Where $\theta$ is polar angle which $P_{i} O$ is polar axis and $P_{i}$ is the pole of polar coordinate.

$\mu\left(r_{x}^{i}\right)$ Can be calculated by the back wave which is detected through the detection point $P_{i}$. So the distribution calculation of target permittivity becomes to compute spatial distribution by line integrals in known space.

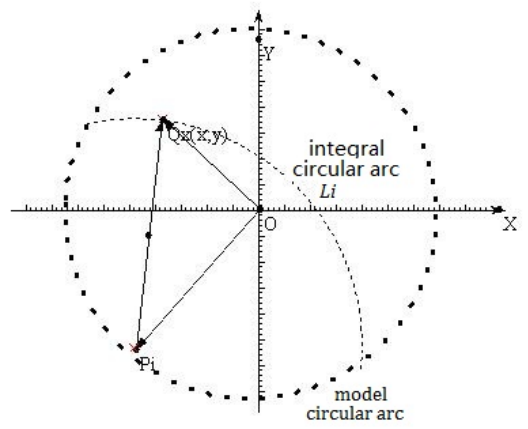

Fig. 1 the relationship between detection point and reflecting interface

The detection of this single test point $P_{i}$ is accumulated along the concentric circular arc direction, and it can be viewed as the projection along the circular arc, which is different from the CT along a linear projection detection. Fig.2 shows schematic diagram of CT detection and microwave detection.

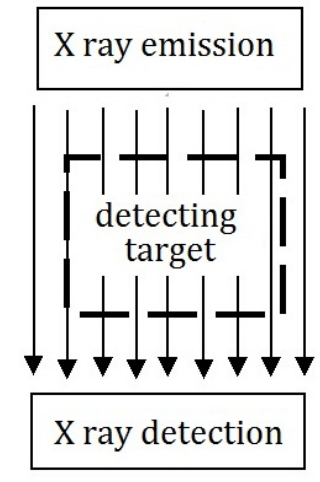

(a)Schematic diagram of CT detection

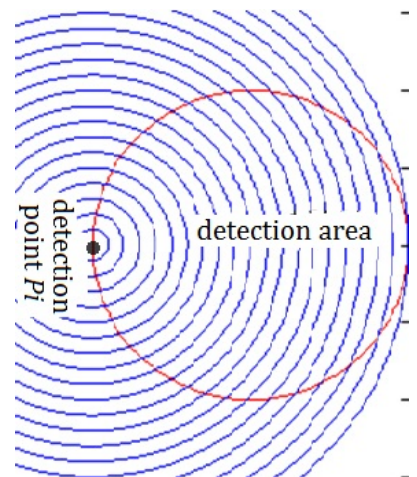

(b) Schematic diagram of microwave detection

In order to calculate the distribution function of the target space, CT uses rotating target method, which is to change the angle of projection ${ }^{[1]}$. Microwave near-field detection uses rotating transceiver antenna method, as shown in figure 3.

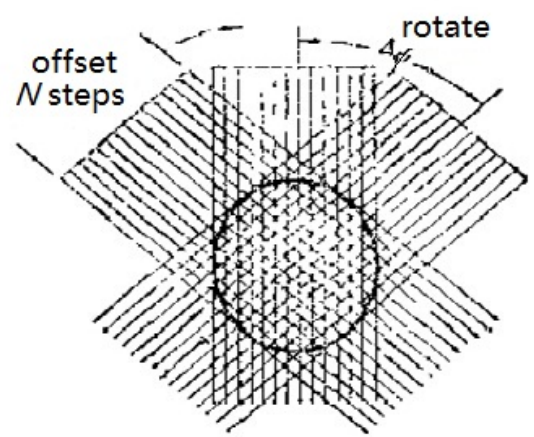

(a)

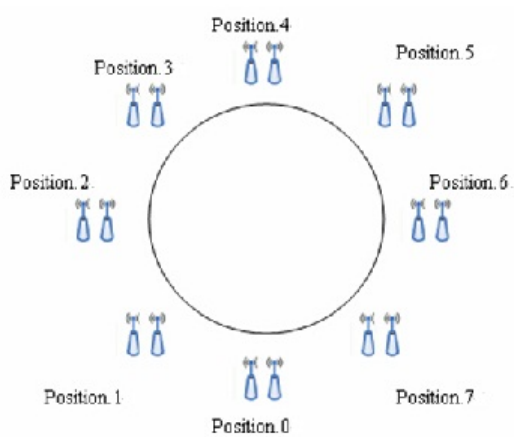

(b) 
Fig. 3 the different detection mode of CT and microwave detection. (a) CT scans with panning and rotating mode. Microwave near field detection places the transceiver antenna as shown in (b).

The target segmentation of two detection methods are entirely different, though they all use rotational detection. In order to facilitate comparison, we consider a detection line through point $\mathrm{Q}$ in target area, which is shown in figure 4.

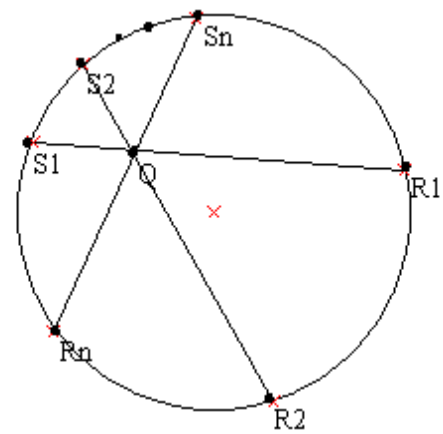

(a)

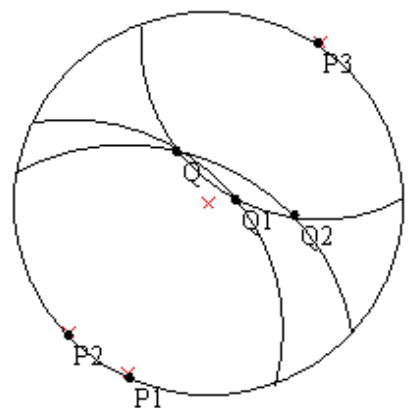

(b)

Fig. 4 The detection difference of CT and microwave near-field detection. (a) In CT, there has only one point of intersection $Q$ in arbitrary two detecting path. (b) In microwave near-field detection, there also have second point of intersection $\left(Q_{1}\right.$ and $\left.Q_{2}\right)$ except $Q$ in arbitrary two detecting path.

For a certain point in the target region, CT can be determined uniquely by two integral paths through the point, which can invert spatial distribution by established linear equations fitted with division of the regional.

For the image reconstruction of $\mathrm{CT}^{[7]}$, we suppose that tomography have $3 * 3$ unit and the attenuation coefficient respectively to each unit are $\mu_{1}$ to $\mu_{9}$. Nine rays, which are completely disjoint, across the tomography. The equation of their line integrals of attenuation coefficient is shown in equation 1 . Where, $P_{1}$ to $P_{9}$ are respectively line integrals of the attenuation coefficient in different rays. $P_{1}$ to $P_{9}$, as a known number, can be confirmed by detector, thus we can get the values of $\mu_{1}$ to $\mu_{9}$. Then we use image representation $\mu_{1}$ to $\mu_{9}$ and generate tomography. That is the reconstruction of CT image.

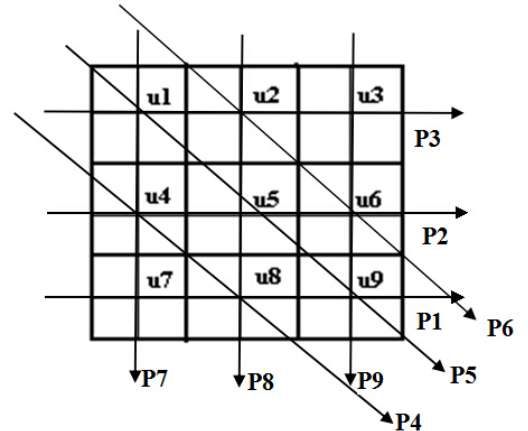

Fig. 5 Schematic diagram of reconstruction of CT images ${ }^{[7]}$

$$
\begin{aligned}
& \mu_{7}+\mu_{8}+\mu_{9}=P_{1} \\
& \mu_{4}+\mu_{5}+\mu_{6}=P_{2} \\
& \mu_{1}+\mu_{2}+\mu_{3}=P_{3} \\
& \mu_{4}+\mu_{8}=P_{4} \\
& \mu_{1}+\mu_{5}+\mu_{9}=P_{5} \\
& \mu_{2}+\mu_{6}=P_{6} \\
& \mu_{1}+\mu_{4}+\mu_{7}=P_{7} \\
& \mu_{2}+\mu_{5}+\mu_{8}=P_{8} \\
& \mu_{3}+\mu_{6}+\mu_{9}=P_{9}
\end{aligned}
$$

\section{[7]}

(1)


(2) The detection wave surface is a sphere, but we reconstruct image only in one plane. That certainly appear many artifacts.

As shown in figure 6, there have a suspicious reflection surface unit $\Gamma$ in detection space. Points $P_{1}$ and $P_{2}$ are two detection points, and the dotted line P1P2 is the inversion plane. $\Gamma$ is the actual suspicious unit, $\Gamma_{1}$ is the corresponding point appearing in the inversion plane using detection point $P_{1}$, and $\Gamma_{2}$ is the corresponding point appearing in the inversion plane using detection point $P_{2}$.

The distance between $\Gamma_{1}$ and $\Gamma_{2}$ is related to the distance between the suspicious unit and inversion plane. In the inversion plane, there form a concentric ring artifacts with the increasing number of points. This is different from the star shaped artifacts produced by CT reconstruction images. Figure 7 is the comparison of artifacts emerged from microwave inversion and CT images.

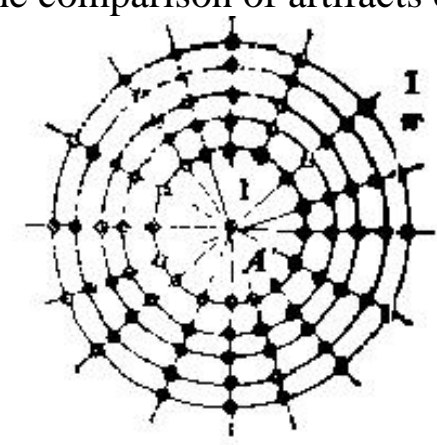

(a)

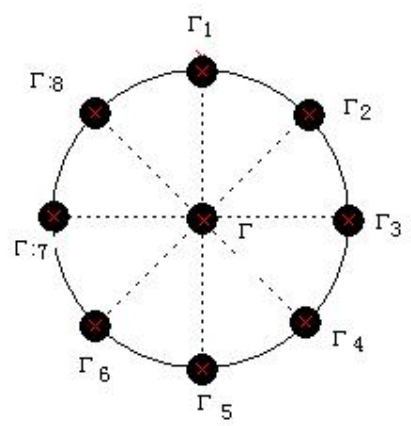

(b)

Fig. 7 The comparison of artifacts emerged from microwave inversion and CT images. (a) The star artifacts of reconstructed by CT. Point A is the real point, and the points distributed with concentric circle are artifacts. (b) The artifacts of reconstructed by microwave near-field reconstruction.

\section{Further work}

Microwave near-field imaging detection method applied to breast cancer detection as a new technology developed in recent years. This article puts a new inversion method to reconstruct microwave near-field image, and give a real image inversion. Then this paper also analyze the reason of artifacts appeared in real microwave near-field detection. Our next step is to further study how to eliminate the artifacts in the imaging process.

\section{References}

[1] American Cancer Society, "Cancer Facts \& Figures 2008," American Cancer Society, Atlanta, 2008

[2] E.C. Fear, P.M. Meaney, and M.A. Stuchly, "Microwaves for breast cancer detection?," IEEE Potentials, vol. 22, no. 1, p. 12-18, 2003.

[3] A. Karellas and S. Vedantham, "Breast cancer imaging: a perspective for the next decade," Med. Phys., vol. 35, no. 11, p. 4878-4897, 2008.

[4] E.C. Fear, "Microwave Imaging of the Breast," Technology in Cancer Research and Treatment, vol. 4, no. 1, p. 69-82, 2005.

[5] M. Lazebnik et al., "A large-scale study of the ultrawideband microwave dielectric properties of normal, benign and malignant breast tissue obtained from cancer surgeries," Phys. Med. Biol., vol. 52, p. 6093-6115, 2007.

[6] Zhifu Tao, Zhongling Han and Meng Yao. The electromagnetic-trait imaging computation of traveling wave method in breast tumor microwave sensor system. Software tools and algorithms for biological systems.[M] p. 489-496

[7] Tiange Zhuang, principle and algorithm of CT, Shanghai Jiao Tong University press, 1992.7 